\title{
The Benefit and Impact of On-Line Tools for Microscopy and Microanalysis Training and Education in Core Facilities.
}

\author{
M. Apperley ${ }^{1}$, P.R. Munroe ${ }^{1}$, T. White $^{2}$, J. Shapter ${ }^{1}$, J. Muhling ${ }^{1}$, L. Soon ${ }^{1}$, S.P. Ringer ${ }^{1}$, E. Grinan ${ }^{1}$, C. \\ Frost ${ }^{1}$ and B. Cribb ${ }^{1}$ \\ 1. Australian Microscopy and Microanalysis Research Facility, Sydney, NSW, 2006, Australia. \\ 2. School of Materials Science and Engineering, Nanyang Technological University, Singapore
}

The Australian Microscopy and Microanalysis Research Facility (AMMRF) is a national grid of equipment, instrumentation and expertise in microscopy and microanalysis that provides nanostructural characterisation capability and services, from widely used optical, electron, X-ray and ion-beam techniques to world-leading flagship platforms. This collaborative facility, comprising a distributed network of microscopy and microanalysis core facilities spread over fourteen institutions, supports more than 3,000 researchers each year of which approximately one-third are "new users" and with indications that this is growing by $10 \%$ per annum.

One of the principal activities of the AMMRF therefore is to provide research training in microscopy and microanalysis. Until recently, much of this training was provided either in the classroom or through one-to-one training at the instrument itself, however, these approaches faced limitations [1].

Firstly, the large number of researchers requiring training places pressures on the core facilities to balance the need to maximise the beam-time of expensive and complex instrumentation for research purposes with that for training new users, who will ultimately perform the research. Prioritising instrument time for research reduces time available for training and vice versa. The specialist staff base within core facilities, that have the requisite knowledge and practical skills to train researchers, is also finite. These are challenges common to facilities offering access to advanced research.

Another common challenge in such facilities is diversity of the student body. In the case of our project the cohort requiring training had a variety of backgrounds and goals: undergraduate students with different educational backgrounds seeking an overview of topic; final year students requiring specific techniques for project work; future career or postgraduate students; and also professional researchers, educators and managers. A more flexible approach to training and education was needed.

To address these challenges and improve the training outcomes of researchers, the AMMRF developed MyScope: Training for Advanced Research [2]. MyScope is an online suite of education tools for teaching and learning in the area of microscopy and microanalysis. It comprises a range of modules developed to sit within the suite: scanning electron microscopy; transmission electron microscopy; scanning probe and atomic force microscopy; microanalysis; confocal microscopy; and X-ray diffraction techniques. The modules in MyScope provide a novel advancement in online training. They contain a number of components including: an interactive questionnaire to allow the user to assess their knowledge, guide choices and tailor the learning environment for flexible learning; also, tailoring capability for academics and trainers; self guided tutorials with videos, animations and glossary to prepare students with knowledge and specialist language; virtual instrument platforms to practice use of instrumentation; and online competency testing to demonstrate readiness for hands-on experience [3]. 
Since it was launched in November 2011 the impact and effectiveness of MyScope has been evaluated. Recent usage data revealed more than 100,000 visits per year had been made to MyScope from over 20 countries with approximately $80 \%$ of those visits unique. Average visit duration was 12:37 min with 15 pages viewed per visit. This data is consistent with focussed learning activity and not mere casual browsing.

The ability of MyScope to address the needs of a diverse cohort of students and researchers was examined with site visit analysis. Data revealed that the largest group of users was graduate students $(45 \%)$. Other roles such as undergraduate students, post-doctoral researchers, facility managers and scientists in industry were represented as significant ( $\sim 10 \%$ each). The prior experience of students was examined with undergraduates, graduates and post-doctoral researchers predominantly seeking training for the first time while users from industry, facility managers and mid career researchers claimed to be experienced microscopists and were using MyScope to learn new skills or set training courses for new researchers in their facilities. While the vast majority of MyScope users were based in universities $(\sim 75 \%)$ there were visits from researchers in government funded laboratories, medical research institutes or industry laboratories.

Data gathered from student questionnaires and via post-use test results indicated that the modules enhanced positive attitudes towards on-line learning platforms, engendered positive attitudes to on-line assessment and enhanced student core knowledge. Use of MyScope prior to small-group practical interaction reduced instruction time without reducing educational outcomes [3].

Evaluation of the effectiveness of MyScope is continuing, however, initial observations have shown that "on column" training time can be reduced by one-third and in some instances by as much as a half. There is anecdotal evidence that indicates when researchers get to the microscope they are much better prepared for the on-column training hence build competency and confidence more quickly. These are positive outcomes since they address the need to maintain high levels of instrument utilisation for research while improving the training throughput of new users.

\section{References}

[1] Cribb, B. et al, Advanced microscopic characterisation through integrated learning tools, Microscopy and Microanalysis, Volume 17, Issue S2, July 2011, pp 870 - 871

[2] www.ammrf.org.au/myscope

[3] Cribb B. et al, MyScope: a national approach to education in advanced microscopic characterization through integrated learning tools, Office for Learning and Teaching, Australian Government Department of Education, ISBN: 978-1925092-03-5

[4] The authors acknowledge funding from the Office for Learning and Teaching, Australian Government Department of Education, CG10-1490. 